\title{
Prevalence of thyroid dysfunction with adequate and excessive iodine intake in Hebei Province, People's Republic of China
}

\author{
Long Tan ${ }^{1}$, Zhongna Sang ${ }^{1}$, Jun Shen ${ }^{2}$, Hua Liu ${ }^{3}$, Wen Chen ${ }^{1}$, Na Zhao', Wei Wei ${ }^{4}$, \\ Guiqin Zhang ${ }^{5}$ and Wanqi Zhang ${ }^{1, *}$ \\ 'Department of Nutrition and Food Science, School of Public Health, Tianjin Medical University, Tianjin 300070, \\ People's Republic of China: ${ }^{2}$ Department of Sanitary Chemistry, School of Public Health, Tianjin Medical University, \\ Tianjin, People's Republic of China: ${ }^{3}$ Centers for Disease Control and Prevention in Cangzhou City, Cangzhou, \\ People's Republic of China: ${ }^{4}$ Chengyang Workstation of Health Supervision and Diseases Control, Qingdao, \\ People's Republic of China: ${ }^{5}$ Department of Clinical Nutrition, Binzhou Medical University Hospital, Binzhou, \\ People's Republic of China
}

Submitted 11 May 2013: Final revision received 25 July 2014: Accepted 3 August 2014: First published online 6 November 2014

\begin{abstract}
Objective: To explore (i) the prevalence of thyroid dysfunction in populations with adequate and excessive iodine intakes and (ii) the effect of iodine exposure on the prevalence of thyroid dysfunction.

Design: Cross-sectional study was conducted in Hebei in 2010. The population was classified as having adequate or excessive iodine intake according to the iodine concentration in drinking water. Demographic information was collected by questionnaire. Levels of serum thyroid hormones, thyroid autoantibodies and iodine in drinking water and urine were measured.

Setting: Villages with adequate or excessive drinking water iodine in Hebei Province, People's Republic of China.

Subjects: A total of 854 men and women aged $20-50$ years who had lived in the surveyed areas for over 5 years, including 348 from the adequate iodine area (AIA) and 506 from the excessive iodine area (EIA).

Results: Median urinary iodine concentration was $185 \mu \mathrm{g} / \mathrm{l}$ in AIA and $1152 \mu \mathrm{g} / \mathrm{l}$ in EIA. The prevalence of thyroid dysfunction in AIA was $10.3 \%$, which included $1.1 \%$ with hypothyroidism and $8.1 \%$ with subclinical hypothyroidism; and $20.6 \%$ in EIA, which included $3.6 \%$ with hypothyroidism and $13.6 \%$ with subclinical hypothyroidism. The positive rates of thyroglobulin antibody were $16 \cdot 1 \%$ in AIA and $11.9 \%$ in EIA; the positive rates of thyroperoxidase antibody were $20.7 \%$ in AIA and $16.4 \%$ in EIA.

Conclusions: Excessive iodine intake may lead to increased prevalence of biochemical thyroid dysfunction, especially biochemical hypothyroidism. This is not related to an increase in prevalence of thyroid antibodies. Women are more susceptible to iodine excess.
\end{abstract}

Keywords
Excessive iodine
Hypothyroidism
Thyroid hormone
Autoantibody
Iodine is an essential trace element for the synthesis of thyroid hormones and thyroxine is also critical for brain development. Iodine deficiency may cause hypothyroidism and results in severe developmental delay in infants and stillbirth in pregnant women ${ }^{(1-3)}$. WHO, UNICEF and the International Council for Control of Iodine Deficiency Disorders (ICCIDD) endorse the policy of iodine supplementation for pregnant and breast-feeding women in iodine-deficient countries where salt iodization is not regulated or is ineffective, in order to improve the nutritional status of iodine ${ }^{(4)}$.

However, iodine deficiency and excessive exposure to iodine due to environmental or artificial factors may lead to thyroid dysfunction ${ }^{(5)}$. Recommended by WHO, ICCIDD and UNICEF, the optimal iodine intake is $150-300 \mu \mathrm{g} / \mathrm{d}$ (median urinary iodine concentration (UIC): 100-199 $\mu \mathrm{g} / \mathrm{l}$ ) ${ }^{(6)}$. Iodine intake exceeding the optimal level will increase the risk of thyroid dysfunction. Chronic iodine intake in the form of iodide is associated with an increase in goitre and subclinical hypothyroidism due to the inhibition of thyroid hormone synthesis ${ }^{(7)}$. Excessive iodine intake can also trigger an immune response, resulting in autoimmune thyroiditis $^{(8)}$, thyroid inflammation, sensitive reaction or acute toxicity ${ }^{(5,7)}$. It has been reported that excessive iodate intake has a minimal impact on thyroid hormones. Hypothyroidism or hyperthyroidism was induced in some 
individuals receiving iodine supplementation. Most abnormalities disappeared after $4-12$ weeks ${ }^{(7,9)}$. However, in our previous study, we found that thyroid dysfunction induced by excessive iodine intake can last for a longer period. The issue regarding the safety of iodine exposure is still controversial and further studies on excessive iodine exposure are highly desired ${ }^{(10)}$.

Universal salt iodization was first introduced in China in 1996, and iodine deficiency disorders are currently under control in most areas ${ }^{(11-13)}$. However, there are still more than ten provinces with waterborne excessive iodine. In these endemic areas, more than 30 million people are exposed to excessive iodine exposure ${ }^{(11-14)}$. With the help of universal salt iodization, iodine intake has increased nationwide $^{(15)}$. However, only a few data have demonstrated the epidemiological characteristics of populations with chronic, more than adequate or excessive iodine intake ${ }^{(15-17)}$.

In order to understand the effect of excessive iodine intake on thyroid health, a cross-sectional survey was conducted in two regions with different iodine intake levels. Excessive iodine in water was $841 \mu \mathrm{g} / \mathrm{l}$ and $394 \mu \mathrm{g} / \mathrm{l}$ in Haixing County; adequate iodine in water was $13 \mu \mathrm{g} / \mathrm{l}$ in the same county, as control. The effect of persistent local excessive iodine intake on the prevalence of thyroid diseases was evaluated.

\section{Methods}

\section{Participants}

Haixing County, a county in Cangzhou City, is located in the south-east of Hebei Province. It is a coastal city with $0 \cdot 22$ million permanent residents and a total area of $960 \mathrm{~km}^{2}$. The study was carried out in April 2010. The criteria for evaluating regions with excessive waterborne iodine, or endemic areas, were according to the national standards (GB/ T19380-2003) of China. According to the records of the local Center for Disease Control and Prevention, of the villages in Haixing County, Zhaogao Village and Fuzhuangzi Village were in the excessive iodine area (EIA) and Xiaoshan Village was in the adequate iodine area (AIA).

All inhabitants living in the research areas completed questionnaires concerning the history and medication of thyroid disease. Comparison of demographic characteristics between questioned inhabitants and examined participants did not reveal any significant differences (Table 1 ). The age of all participants was in the range of $20-50$ years and these people had lived in the surveyed areas for over 5 years.

Research protocols were approved by the Medical Ethics Committee of Tianjin Medical University. All participants provided written informed consent after the research protocols were carefully explained to them.

\section{Sample collection}

Blood samples were collected from all participants during 08.00-12.00 hours. Serum samples were separated and stored at $-20^{\circ} \mathrm{C}$ until analysis. Casual morning (between 08.00 and 10.30 hours) urine samples were collected from all participants in clean plastic specimen containers and stored at $4^{\circ} \mathrm{C}$. The drinking water of all three villages was extracted underground and supplied by the local government through a pipe network system. Every village owns its particular underground water source. Peripheral drinking water samples were collected in five different positions such as south, north, east, west and middle of every surveyed village. Two drinking water samples were sampled from two different families in each position. The salt was not analysed because the iodized salt was not regulated in these counties.

\section{Concentrations of iodine in drinking water and urine}

The concentrations of iodine in drinking water and urine were measured by the national standard method as described previously ${ }^{(10)}$.

\section{Thyroid hormones and autoantibodies}

The contents of free thyroxine $\left(\mathrm{FT}_{4}\right)$, free triiodothyronine $\left(\mathrm{FT}_{3}\right)$ and thyroid-stimulating hormone (TSH) in serum from all participants were measured by an automated chemiluminescent immunoassay using diagnostic kits from Bayer Health Care, Siemens. The normal ranges were $11.5-23.5 \mathrm{pmol} / 1$ for $\mathrm{FT}_{4}, 3.5-6.5 \mathrm{pmol} / 1$ for $\mathrm{FT}_{3}$ and $0 \cdot 3-5 \mathrm{mIU} / 1$ for $\mathrm{TSH}^{(10)}$.

The contents of thyroperoxidase antibody (TPOAb) and thyroglobulin antibody (TgAb) in serum were measured by RIA kits from Beijing North Institution. TPOAb and TgAb assays were completed using the standard reference materials that were produced by Tianjin Nine Tripods Medical \& Biological Co. $\operatorname{Ltd}^{(10)}$.

\section{Diagnostic criteria}

The diagnostic criteria for thyroid diseases were as follows: hypothyroidism (TSH $>5.0 \mathrm{mIU} / 1$ and $\left.\mathrm{FT}_{4}<11.5 \mathrm{pmol} / \mathrm{l}\right)$; subclinical hypothyroidism (TSH $>5.0 \mathrm{mIU} / 1,11.5 \mathrm{pmol} / \mathrm{l}$ $<\mathrm{FT}_{4}<23.5 \mathrm{pmol} / 1$ and $\left.3.5 \mathrm{pmol} / 1<\mathrm{FT}_{3}<6.5 \mathrm{pmol} / \mathrm{l}\right)$; hyperthyroidism $\left(\mathrm{TSH}<0.3 \mathrm{mIU} / 1\right.$, and $\mathrm{FT}_{4}>23.5 \mathrm{pmol} / 1$ or $\left.\quad \mathrm{FT}_{3}>6.5 \mathrm{pmol} / \mathrm{l}\right) ; \quad$ subclinical hyperthyroidism $\left(\mathrm{TSH}<0.3 \mathrm{mIU} / 1, \quad 11.5 \mathrm{pmol} / 1<\mathrm{FT}_{4}<23.5 \mathrm{pmol} / 1 \quad\right.$ and $\left.3.5 \mathrm{pmol} / 1<\mathrm{FT}_{3}<6.5 \mathrm{pmol} / \mathrm{l}\right)^{(10)}$.

\section{Statistical analysis}

All tests were two-tailed and a statistically significant difference was considered at $P<0 \cdot 05$. Quantitative data were expressed as mean and standard deviation, except the data on urinary iodine and serum TSH that were expressed as median and interquartile range (IQR; 25 th-75th percentile). $\mathrm{R} \times \mathrm{C}$ categorical data were analysed by the $\chi^{2}$ test. The $2 \times 2$ table was analysed by Fisher's exact test (without Bonferroni correction). Differences between the two areas for age, body weight, height, iodine intake, and serum $\mathrm{FT}_{3}, \mathrm{FT}_{4}$ and $\mathrm{TSH}$ contents were tested by using independent sample $t$ tests, and UIC was analysed through 
the Kruskal-Wallis test. Statistical analyses were conducted using the SPSS statistical software package version 13.0.

\section{Results}

\section{Participants}

The concentration of iodine in drinking water from Fuzhuangzi Village, Zhaogao Village and Xiaoshan Village was 841,394 and $13 \mu \mathrm{g} / \mathrm{l}$, respectively. After being screened by questionnaires, a total of 854 inhabitants, including 348 (112 males and 236 females) in AIA and 506 (185 males and 321 females) in EIA, were investigated. The two populations were similar with respect to age, sex, economic status, accessibility to health care and smoking rate.

\section{Concentration of urinary iodine}

Median UIC was 185 (IQR 147-240) $\mu \mathrm{g} / \mathrm{l}$ in AIA and 1152 (IQR 753-1539) $\mu \mathrm{g} / \mathrm{l}$ in EIA. The distribution of the individual values within categories is reported in Table 1.

\section{Thyroid bormones and autoantibodies}

Mean serum $\mathrm{FT}_{3}$ concentration was significantly lower and mean serum TSH concentration was significantly higher in EIA than in AIA. Mean serum $\mathrm{FT}_{4}$ was similar in both areas (Table 2).

The total positive rates of TgAb and TPOAb were not statistically different between EIA and AIA (16.1\% v. $11.9 \%$ for TgAb and $20.7 \% v .16 .4 \%$ for TPOAb). However, there was a significant difference in autoantibodies between genders, with the positive rates of autoantibodies in females being significantly higher than in males. Specifically, in AIA, the positive rate of TgAb in females was $6 \cdot 1$-fold higher than in males $\left(22.0 \% v \cdot 3 \cdot 6 \% ; \chi^{2}=19 \cdot 174, P=0.000\right)$ and the positive rate of TPOAb was $2 \cdot 4$-fold higher $(25 \cdot 4 \% v$. $\left.10.7 \% ; \chi^{2}=10 \cdot 015, P=0 \cdot 002\right)$. In EIA, the positive rate of TgAb in females was 2.9-fold higher than that in males $\left(15 \cdot 6 \% v \cdot 5 \cdot 4 \% ; \chi^{2}=11 \cdot 616, P=0.001\right)$ and the positive rate of TPOAb was $1 \cdot 8$-fold higher $\left(19 \cdot 6 \% v \cdot 10 \cdot 8 \% ; \chi^{2}=6 \cdot 651\right.$, $P=0.010$ ). However, the positive rates of autoantibodies were not significantly different between AIA and EIA when comparing by gender (Table 3).

The percentage of positive thyroid antibodies (TgAb and TPOAb) was similar in both areas (with adequate iodine and with excessive iodine). This percentage was at least twice greater in women than in men (Table 3 ).

\section{Prevalence of thyroid dysfunction}

Thyroid dysfunction was two times more frequent in EIA than in AIA $(20 \cdot 6 \% v \cdot 10 \cdot 3 \%)$. Frequency of subclinical hypothyroidism (elevated TSH and normal $\mathrm{FT}_{4} ; 13 \cdot 6 \% v$. $9.0 \%$ ) and subclinical hyperthyroidism (decreased TSH and normal $\mathrm{FT}_{4} ; 2.2 \% \quad v .0 \%$ ) differed significantly between both areas. Hypothyroidism (decreased TSH and elevated $\mathrm{FT}_{4} ; 3.6 \%$ v. $1.3 \%$ ), but not hyperthyroidism (decreased $\mathrm{TSH}$ and elevated $\mathrm{FT}_{4}$ ), was more frequent (not statistically significant) in EIA than in AIA. In men, the proportion of thyroid dysfunction was similar in AIA and EIA. By contrast, it was more than two times greater in females from EIA than in females from AIA (Table 4). As a result, there was no significant difference in thyroid dysfunction frequency by gender in AIA, but a highly significant difference in EIA (Fisher's exact test, $P=2 \cdot 3 \times 10^{-4}$ ).

More than $10 \%$ of euthyroid participants showed at least one positive thyroid antibody test and $8.1 \%$ were positive for both thyroid antibodies. In the hypothyroid participants, this percentage of positive anti-thyroid test was more or less double (Table 5). Due to the small number of

Table 1 Urinary iodine concentration and distribution in men and women aged 20-50 years who had adequate or excessive drinking water iodine, Hebei Province, People's Republic of China, 2010

\begin{tabular}{|c|c|c|c|c|c|c|}
\hline & \multicolumn{2}{|c|}{ UIC $(\mu \mathrm{g} / \mathrm{l})$} & \multicolumn{2}{|c|}{ UIC $<100 \mu \mathrm{g} / \mathrm{l}$} & \multicolumn{2}{|c|}{$\mathrm{UIC} \geq 300 \mu \mathrm{g} / \mathrm{l}$} \\
\hline & Median & IQR & $n$ & $\%$ & $n$ & $\%$ \\
\hline $\begin{array}{l}\text { AIA }(n 348) \\
\text { EIA }(n 506)\end{array}$ & $\begin{array}{r}185 \\
1152\end{array}$ & $\begin{array}{l}147-240 \\
753-1539\end{array}$ & $\begin{array}{r}45 \\
0\end{array}$ & $\begin{array}{r}12.9 \\
0.0\end{array}$ & $\begin{array}{r}87 \\
471\end{array}$ & $\begin{array}{l}25.0 \\
93.1\end{array}$ \\
\hline Comparison: AIA v. EIA & \multicolumn{2}{|c|}{ Wilcoxon: $P<0.001$} & \multicolumn{2}{|c|}{$x^{2} . P<0.001$} & \multicolumn{2}{|c|}{$x^{2}: P<0.001$} \\
\hline
\end{tabular}

UIC, urinary iodine concentration; IQR, interquartile range; AIA, adequate iodine area; EIA, excessive iodine area; Wilcoxon, Wilcoxon rank sum test; $X^{2}, \chi^{2}$ test.

Table 2 Levels of thyroid hormones in men and women aged 20-50 years who had adequate or excessive drinking water iodine, Hebei Province, People's Republic of China, 2010

\begin{tabular}{|c|c|c|c|c|c|c|}
\hline & \multicolumn{2}{|c|}{ Serum $\mathrm{FT}_{4}(\mathrm{pmol} / \mathrm{l})$} & \multicolumn{2}{|c|}{ Serum $\mathrm{FT}_{3}(\mathrm{pmol} / \mathrm{l})$} & \multicolumn{2}{|c|}{ Serum TSH (mlU/l) } \\
\hline & Mean & SD & Mean & SD & Median & IQR \\
\hline AIA (n 348) & 14.75 & $2 \cdot 71$ & 4.93 & 0.60 & 2.40 & $1.58-3.56$ \\
\hline EIA $(n 506)$ & 14.68 & 3.35 & 4.77 & 0.94 & $2 \cdot 71$ & $1.73-4.56$ \\
\hline Comparison: AIA v. EIA & \multicolumn{2}{|c|}{$t$ test: $P=0.729$} & \multicolumn{2}{|c|}{$t$ test: $P=0.003$} & \multicolumn{2}{|c|}{ Wilcoxon: $P=0.002$} \\
\hline
\end{tabular}

$\mathrm{FT}_{4}$, free thyroxine; $\mathrm{FT}_{3}$, free triiodothyronine; $\mathrm{TSH}$, thyroid-stimulating hormone; IQR, interquartile range; AIA, adequate iodine area; EIA, excessive iodine area; $t$ test, Student's $t$ test; Wilcoxon, Wilcoxon rank sum test. 
dysfunction in China, a cross-sectional investigation in three areas with adequate or excessive iodine intake was carried out. Hebei Province is located downstream of the Yellow River. The EIA in Hebei mainly were scattered around the estuary of the Yellow River. The high iodine concentration in drinking water is an important source of iodine intake by the inhabitants. The water of the surveyed villages is extracted independently underground and supplied to the inhabitants through the pipe network system. The depth of the underground water source is different, from less than $100 \mathrm{~m}$ to more than $400 \mathrm{~m}$. So the iodine concentration is different according to the depth of source water.

The results showed that the serum levels of both $\mathrm{FT}_{3}$ and TSH were affected by the excessive iodine intake. The average level of $\mathrm{FT}_{3}$ in EIA revealed a slight decrease when compared with that in AIA, while the level of TSH exhibited a slight increase in EIA. The results suggest a link between increased iodine intake and hypothyroidism. Similar results have been reported in a randomized controlled trial in older people ${ }^{(9)}$. With a supplementation of $80 \mu \mathrm{g}$ or $>50 \mathrm{mg}$ iodate daily for 8 weeks, ten of fortythree participants exposed to excessive iodate showed hypothyroidism at the end of the trial. A 5-year cohort study on the prevalence of thyroid dysfunction in populations with different iodine intakes such as mildly deficient (median UIC: $84 \mu \mathrm{g} / \mathrm{l}$ ), more than adequate (median UIC: $243 \mu \mathrm{g} / \mathrm{l}$ ) and excessive (median UIC: $651 \mu \mathrm{g} / \mathrm{l}$ ) in China has been completed ${ }^{(15)}$. An increase in the prevalence of overt hypothyroidism, subclinical hypothyroidism and autoimmune thyroiditis with increasing iodine intake was observed. Another follow-up study in three rural Chinese communities with mild to borderline deficiency, more than adequate and excessive iodine intake, respectively, has been carried out ${ }^{(18)}$. On the contrary, a follow-up examination has been provided to these inhabitants for 13 years and the results demonstrated that chronic excessive iodine intake does not apparently increase the risk of autoimmune hyperthyroidism. In the present study, although we did not detect a significant difference in the prevalence of hyperthyroidism between AIA and EIA, we found that the prevalence of subclinical hyperthyroidism in EIA was significantly higher than that in AIA. No subclinical hyperthyroidism case was observed in AIA, and eleven inhabitants living in EIA were diagnosed as subclinical hyperthyroidism in our research.

The thyroid dysfunction spectrum of AIA and EIA was also similar. It has been reported that the prevalence of subclinical hyperthyroidism and hyperthyroidism may be increased among people with more than adequate and excessive iodine intake ${ }^{(15)}$. Similarly, high iodine intake may trigger and aggravate autoimmune thyroiditis, thus increasing the risk of clinical hypothyroidism. Moreover, the titres of autoantibodies, TgAb and TPOAb, in females are sensitive to iodine exposure. Higher levels of autoantibodies in females from AIA and EIA were observed when compared with those in males. In addition, a casecontrol study has reported that excessive iodine intake may trigger thyroid autoimmunity and hypothyroidism among women ${ }^{(19)}$. These results indicate that women may be a susceptible population and even a high-risk population to iodine exposure.

Our data did not provide direct evidence that excessive iodine exposure could increase the risk of thyroid autoimmunity. However, the results showed a relationship between the positive rate of autoantibodies and the severity of hypothyroidism. A higher positive rate of autoantibodies was observed in hypothyroidism compared with that in subclinical hypothyroidism and euthyroidism. In Li et al.'s study, only an increased positive rate of $\mathrm{TgAb}$ with increased iodine intake was reported and individuals with a positive rate of TPOAb and/or TgAb had more frequent development of thyroid dysfunction ${ }^{(20)}$. These results suggest that autoantibodies such as TgAb and TPOAb are effective parameters associated with the development of thyroid dysfunction, especially hypothyroidism.

\section{Conclusion}

In conclusion, excessive iodine intake (iodine concentrations in water were $394 \mu \mathrm{g} / \mathrm{l}$ and $841 \mu \mathrm{g} / \mathrm{l}$, and median UIC was $1152 \mu \mathrm{g} / \mathrm{l}$, which is larger than $300 \mu \mathrm{g} / \mathrm{l}$ ) is not safe. Excessive iodine exposure may be a risk factor for an increased prevalence of biochemical thyroid dysfunction, especially biochemical hypothyroidism. The iodine supplementation should be tailored according to the level of iodine intake. Women may be a susceptible population to excessive iodine exposure.

\section{Acknowledgements}

Financial support: This work was supported by the National Natural Science Foundation of China (grant numbers 81273057, 30972465 and 81202201). The funder had no role in the design, analysis or writing of this article. Conflict of interest: None. Authorship: L.T. collected the data and conducted manuscript draft writing; Z.S. conducted data collection and analysis; J.S. and H.L. supervised the survey; W.C., N.Z., W.W. and G.Z. collected the data and completed the laboratory determinations; W.Z. developed the study hypothesis and instructed the data analysis and editing of the manuscript. L.T. and Z.S. contributed to this project equally. Etbics of buman subject participation: Research protocols were approved by the Medical Ethics Committee of Tianjin Medical University.

\section{References}

1. World Health Organization, UNICEF \& International Council for Control of Iodine Deficiency Disorders (2007) Assessment of Iodine Deficiency Disorders and Monitoring 
their Elimination. A Guide for Programme Managers, 3rd ed. Geneva: WHO.

2. Zimmermann MB, Jooste PL \& Pandav CS (2008) Iodinedeficiency disorders. Lancet 372, 1251-1262.

3. Takeuchi T, Kamasaki H, Yoto Y et al. (2012) Investigation of iodine deficient state and iodine supplementation in patients with severe motor and intellectual disabilities on long-term total enteral nutrition. Endocr J 59, 697-703.

4. Andersson M, de Benoist B, Delange F et al. (2007) Prevention and control of iodine deficiency in pregnant and lactating women and in children less than 2-years-old: conclusions and recommendations of the Technical Consultation. Public Health Nutr 10, 1606-1611.

5. Pennington JA (1990) A review of iodine toxicity reports. J Am Diet Assoc 90, 1571-1581.

6. World Health Organization, International Council for Control of Iodine Deficiency Disorders \& UNICEF (2001) Assessment of Iodine Deficiency Disorders and Monitoring their Elimination. A Guide for Programme Managers, 2nd ed. Geneva: WHO.

7. Stanbury JB, Ermans AE, Bourdoux P et al. (1998) Iodineinduced hyperthyroidism: occurrence and epidemiology. Thyroid 8, 83-100.

8. Zimmermann MB (2008) Iodine requirements and the risks and benefits of correcting iodine deficiency in populations. J Trace Elem Med Biol 22, 81-92.

9. Thomson CD, Campbell JM, Miller J et al. (2011) Minimal impact of excess iodate intake on thyroid hormones and selenium status in older New Zealanders. Eur J Endocrinol 165, 745-752.

10. Sang Z, Wang PP, Yao Z et al. (2012) Exploration of the safe upper level of iodine intake in euthyroid Chinese adults: a randomized double-blind trial. Am J Clin Nutr 95, 367-373.
11. Chen ZP, Yan Y \& Shu YQ (2001) Analysis of iodine intake levels and safe range of iodine intake after universal salt iodization in China. Chin J Control Endem Dis 16, 185-188.

12. Liu Y, Liu SJ, Zhang SB et al. (2003) National surveillance of urinary iodine concentrations of children aged 8 to 10 years: an analysis of the results. Chin J Endemiol 22, 380.

13. Shen HM, Zhang SB, Liu SJ et al. (2007) Study on the geographic distribution of national high water iodine areas and the contours of water iodine in high iodine areas. Chin J Endemiol 26, 658-661.

14. Zhao J, Wang P, Shang L et al. (2000) Endemic goiter associated with high iodine intake. Am J Public Health 90, 1633-1635.

15. Teng W, Shan Z, Teng X et al. (2006) Effect of iodine intake on thyroid diseases in China. N Engl J Med 354, 2783-2793.

16. Wu Y, Li X, Chang S et al. (2012) Variable iodine intake persists in the context of universal salt iodization in China. J Nutr 142, 1728-1734.

17. Li C, Guan H, Teng X et al. (2011) An epidemiological study of the serum thyrotropin reference range and factors that influence serum thyrotropin levels in iodine sufficient areas of China. Endocr J 58, 995-1002.

18. Yang F, Shan Z, Teng X et al. (2007) Chronic iodine excess does not increase the incidence of hyperthyroidism: a prospective community-based epidemiological survey in China. Eur J Endocrinol 156, 403-408.

19. Alsayed A, Gad AM, Abdel-Baset $\mathrm{H}$ et al. (2008) Excess urinary iodine is associated with autoimmune subclinical hypothyroidism among Egyptian women. Endocr J 55, 601-605.

20. Li Y, Teng D, Shan Z et al. (2008) Antithyroperoxidase and antithyroglobulin antibodies in a five-year follow-up survey of populations with different iodine intakes. J Clin Endocrinol Metab 93, 1751-1757. 